\title{
REGIONS OF VARIABILITY FOR JANOWSKI FUNCTIONS
}

\author{
M. RAZA, W. UL-HAQ, AND S. NOREEN \\ Received 20 September, 2014
}

Abstract. Let $A \in \mathbb{C}, B \in[-1,0)$. Then $P[A, B]$ denotes the class of analytic functions $p$ in the open unit disk with $p(0)=1$ such that

$$
p(z)=\frac{1+A w(z)}{1+A w(z)},
$$

where $w(0)=0$ and $|w(z)|<1$. In this article we find the regions of variability $V\left(z_{0}, \lambda\right)$ for $\int_{0}^{z_{0}} p(\rho) d \rho$ when $p$ ranges over the class $P_{\lambda}[A, B]$ defined as

$$
P_{\lambda}[A, B]=\left\{p \in P[A, B]: p^{\prime}(0)=(A-B) \lambda\right\}
$$

for any fixed $z_{0} \in E$ and $\lambda \in \bar{E}$. As a consequence, the regions of variability are also illustrated graphically for different sets of parameters.

2010 Mathematics Subject Classification: 30C45; 30C55; 30C80

Keywords: region of variability, Janowski functions

\section{INTRODUCTION}

Let $A$ denote the class of analytic functions of the form

$$
f(z)=z+\sum_{n=2}^{\infty} a_{n} z^{n}
$$

in the open unit disk $E=\{z:|z|<1\}$. Also Consider $A$ as a topological vector space endowed with the topology of uniform convergence over a compact subsets of $E$. Also let $\mathscr{B}$ denote the class of analytic functions $w$ in $E$ such that $|w(z)|<1$ and $w(0)=0$. A function $f$ is said to be subordinate to a function $g$ written as $f \prec g$, if there exists a Schwarz function $w$ with $w(0)=0$ and $|w(z)|<1$ such that $f(z)=$ $g(w(z))$. In particular if $g$ is univalent in $E$, then $f(0)=g(0)$ and $f(E) \subset g(E)$. Let $P[A, B]$ denote the class of analytic functions $p$ such that $p(0)=1$ and

$$
p(z)=\frac{1+A w(z)}{1+B w(z)}, w \in B, z \in E,
$$

W.Ul-Haq wishes to acknowledge Deanship of Scientific Research (DSR) Majmaah University for financial and technical support under the project "Certain new trends in GFT". 
where $A \in \mathbb{C}, B \in[-1,0)$ with $A \neq B$. Note that $P[1,-1]=P$.

Let $p \in P[A, B]$. Then using the Herglotz representation, there exists a unique positive unit measure $\mu$ in $(-\pi, \pi]$ such that

$$
p(z)=\int_{-\pi}^{\pi} \frac{1+A e^{i t} z}{1+B e^{i t} z} d \mu(t), z \in E .
$$

It can easily be seen from (1.2) that

$$
w_{p}(z)=\frac{p(z)-1}{A-B p(z)}, z \in E,
$$

and conversely, we have

$$
p^{\prime}(0)=(A-B) w_{p}^{\prime}(0)
$$

Let $p \in P[A, B]$. Then by using classical Schwarz lemma, that is $\left|w_{p}^{\prime}(0)\right| \leq 1$ (see [1],) we obtain

$$
p^{\prime}(0)=(A-B) \lambda
$$

for some $\lambda \in \bar{E}$. By using (1.4) one can compute

$$
\frac{w_{p}^{\prime \prime}(0)}{2}=\frac{p^{\prime \prime}(0)}{2(A-B)}+B \lambda^{2}
$$

Now, if we let

$$
g(z)=\left\{\begin{array}{cc}
\frac{\frac{w_{p}(z)}{z}-\lambda}{z-\bar{\lambda} \frac{w_{p}(z)}{z}}, & |\lambda|<1 \\
0 & |\lambda|=1
\end{array}\right.
$$

This implies that

$$
g^{\prime}(0)=\left\{\begin{array}{cc}
\left.\frac{1}{1-|\lambda|^{2}}\left(\frac{w_{p}(z)}{z}\right)^{\prime}\right|_{z=0}=\frac{1}{1-|\lambda|^{2}} \frac{w_{p}^{\prime \prime}(z)}{2}, & |\lambda|<1, \\
0 & |\lambda|=1 .
\end{array}\right.
$$

By Schwarz lemma for $|\lambda|<1$, we see that $|g(z)| \leq|z|$ and $\left|g^{\prime}(0)\right| \leq 1$. Equality is attained in both cases if $g(z)=e^{i \alpha} z$ for some $\alpha \in \mathbb{R}$. Now the condition $\left|g^{\prime}(0)\right| \leq 1$ shows that there exists an $a \in \bar{E}$ such that $g^{\prime}(0)=a$. Thus, we have

$$
p^{\prime \prime}(0)=2(A-B)\left[\left(1-|\lambda|^{2}\right) a-B \lambda^{2}\right] \text {. }
$$

Consequently for $\lambda \in \bar{E}$ and $z_{0} \in E$, we have $V\left(z_{0}, \lambda\right)$ for $\int_{0}^{z_{0}} p(\rho) d \rho$ when $p$ ranges over the class $P_{\lambda}[A, B]$ defined as

$$
P_{\lambda}[A, B]=\left\{p \in P[A, B]: p^{\prime}(0)=(A-B) \lambda\right\}
$$

and

$$
V_{\lambda}\left(z_{0}, A, B\right)=\left\{\int_{0}^{z_{0}} p(\rho) d \rho, \quad p \in P_{\lambda}[A, B]\right\} .
$$

For related study we refer to [2-9] and the references therein. The aim of this paper is to investigate explicitly the region of variability $V_{\lambda}\left(z_{0}, A, B\right)$ for the class $P_{\lambda}[A, B]$. 


\section{BASIC PROPERTIES OF $V_{\lambda}\left(z_{0}, A, B\right)$}

Proposition 1. (i) $V_{\lambda}\left(z_{0}, A, B\right)$ is a compact subset of $\mathbb{C}$.

(ii) $V_{\lambda}\left(z_{0}, A, B\right)$ is convex subset of $\mathbb{C}$.

(iii) If $|\lambda|=1$ or $z_{0}=0$, then $V_{\lambda}\left(z_{0}, A, B\right)=\left\{z_{0}-\frac{A-B}{B}\left(z_{0}+\frac{1}{B \lambda} \log \left(1+B \lambda z_{0}\right)\right)\right\}$ and if $|\lambda|<1$ and $z_{0} \neq 0$, then $\left\{z_{0}-\frac{A-B}{B}\left(z_{0}+\frac{1}{B \lambda} \log \left(1+B \lambda z_{0}\right)\right)\right\}$ is the interior point of the set $V_{\lambda}\left(z_{0}, A, B\right)$.

Proof. (i) Since $P_{\lambda}[A, B]$ is a compact subset of $\mathbb{C}$, therefore $V_{\lambda}\left(z_{0}, A, B\right)$ is also compact.

(ii) Let $p_{1}, p_{2} \in P_{\lambda}[A, B]$. Then

$$
p(z)=(1-t) p_{1}(z)+t p_{2}(z), t \in[0,1]
$$

is also in $P_{\lambda}[A, B]$, therefore $V_{\lambda}\left(z_{0}, A, B\right)$ is convex.

(iii) Since if $|\lambda|=\left|w_{f}^{\prime}(0)\right|=1$, then from Schwarz lemma, we obtain $w_{f}(z)=\lambda z$, which yields $p(z)=\frac{1+\lambda A z}{1+\lambda B z}$. This implies that

$$
p(z)=1+\left(\frac{A-B}{B}\right)\left\{1-\frac{1}{1+B \lambda z}\right\} .
$$

Therefore

$$
V_{\lambda}\left(z_{0}, A, B\right)=\int_{0}^{z_{0}} p(\rho) d \rho=\left\{z_{0}-\frac{A-B}{B}\left(z_{0}+\frac{1}{B \lambda} \log \left(1+B \lambda z_{0}\right)\right)\right\} .
$$

This also trivially holds true when $z_{0}=0$. For $\lambda \in E$ and $\alpha \in \bar{E}$, set

$$
\begin{gathered}
\delta(z, \lambda)=\frac{z+\lambda}{1+\bar{\lambda} z} \\
\int_{0}^{z} H_{a, \lambda}(\rho) d \rho=\int_{0}^{z} \frac{1+A \rho \delta(a \rho, \lambda)}{1+B \rho \delta(a \rho, \lambda)} d \rho, z \in E .
\end{gathered}
$$

Then $\int_{0}^{z} H_{a, \lambda}(\rho) d \rho$ is in $P_{\lambda}[A, B]$ and $w_{f}(z)=z \delta(a z, \lambda)$. For fixed $\lambda \in E$ and $z_{0} \in E \backslash\{0\}$ the function

$$
E \ni a \mapsto \int_{0}^{z_{0}} H_{a, \lambda}(\rho) d \rho=\int_{0}^{z_{0}} \frac{1+(\bar{\lambda} a+A \lambda) \rho+A a \rho^{2}}{1+(\bar{\lambda} a+B \lambda) \rho+B a \rho^{2}} d \rho
$$

is a non-constant analytic function of $a \in E$, and therefore is an open mapping. Hence $\int_{0}^{z_{0}} H_{0, \lambda}(\rho) d \rho=\left\{z_{0}-\frac{A-B}{B}\left(z_{0}+\frac{1}{B \lambda} \log \left(1+B \lambda z_{0}\right)\right)\right\}$ is an interior point of $\left\{\int_{0}^{z_{0}} H_{a, \lambda}(\rho) d \rho: a \in E\right\} \subset V_{\lambda}\left[z_{0}, A, B\right]$.

Keeping in view the above proposition, it is sufficient to find $V_{\lambda}\left[z_{0}, A, B\right]$ for $0 \leq \lambda<1$ and $z_{0} \in E \backslash\{0\}$. 


\section{MAin Results}

In this section, we state and prove some results which are needed in the proof of our main theorems.

Proposition 2. For $p \in P_{\lambda}[A, B]$, we have

$$
|p(z)-q(z, \lambda)| \leq r(z, \lambda), z \in E, \lambda \in \bar{E},
$$

where

$$
\begin{aligned}
q(z, \lambda) & =\frac{(1+\lambda A z)(1+B \bar{\lambda} \bar{z})-|z|^{2}(\lambda+B \bar{z})(\bar{\lambda}+A z)}{1-B^{2}|z|^{4}+2 B\left(1-|z|^{2}\right) \Re(\lambda z)+|\lambda|^{2}|z|^{2}\left(B^{2}-1\right)}, \\
r(z, \lambda) & =\frac{|A-B|\left(1-|\lambda|^{2}\right)|z|^{2}}{1-B^{2}|z|^{4}+2 B\left(1-|z|^{2}\right) \Re(\lambda z)+|\lambda|^{2}|z|^{2}\left(B^{2}-1\right)} .
\end{aligned}
$$

The inequality is sharp for $z_{0} \in E \backslash\{0\}$ if and only if $f(z)=H_{e^{i \theta}, \lambda}(z)$ for some $\theta \in \mathbb{R}$.

Proof. Let $p \in P_{\lambda}[A, B]$. Then there exists $w_{p} \in \mathscr{B}$ such that

$$
\left|\frac{\frac{w_{p}(z)}{z}-\lambda}{1-\bar{\lambda} \frac{w_{p}(z)}{z}}\right| \leq|z|
$$

From (1.4) this can be written equivalently as

$$
\left|\frac{p(z)-b(z, \lambda)}{p(z)+c(z, \lambda)}\right| \leq|z||\tau(z, \lambda)|,
$$

where

$$
b(z, \lambda)=\frac{1+\lambda A z}{1+\lambda B z}, c(z, \lambda)=-\frac{\bar{\lambda}+A z}{B z+\bar{\lambda}}, \tau(z, \lambda)=\frac{\bar{\lambda}+B z}{1+\lambda B z} .
$$

Simple computations show that the inequality (3.4) can be written as

$$
\left|p(z)-\frac{b(z, \lambda)+|z|^{2}|\tau(z, \lambda)|^{2} c(z, \lambda)}{1-|z|^{2}|\tau(z, \lambda)|^{2}}\right| \leq \frac{|z||\tau(z, \lambda)||b(z, \lambda)+c(z, \lambda)|}{1-|z|^{2}|\tau(z, \lambda)|^{2}} .
$$

Now we have

$$
\begin{aligned}
& 1-|z|^{2}|\tau(z, \lambda)|^{2}=\frac{1-B^{2}|z|^{4}+2 B\left(1-|z|^{2}\right) \Re(\lambda z)+|\lambda|^{2}|z|^{2}\left(B^{2}-1\right)}{|1+\lambda z B|^{2}}, \\
& b(z, \lambda)+c(z, \lambda)=\frac{(B-A)\left(1-|\lambda|^{2}\right) z}{(1+\lambda B z)(B z+\bar{\lambda})}, \\
& b(z, \lambda)+|z|^{2}|\tau(z, \lambda)|^{2} c(z, \lambda)
\end{aligned}
$$




$$
=\frac{(1+\lambda A z)(1+B \bar{\lambda} \bar{z})}{|1+\lambda z B|^{2}}-\frac{|z|^{2}(\lambda+B \bar{z})(\bar{\lambda}+A z)}{|1+\lambda z B|^{2}} .
$$

By simple calculations, we obtain

$$
\begin{aligned}
& q(z, \lambda)=\frac{b(z, \lambda)+|z|^{2}|\tau(z, \lambda)|^{2} c(z, \lambda)}{1-|z|^{2}|\tau(z, \lambda)|^{2}}, \\
& r(z, \lambda)=\frac{|z||\tau(z, \lambda)||b(z, \lambda)+c(z, \lambda)|}{1-|z|^{2}|\tau(z, \lambda)|^{2}} .
\end{aligned}
$$

All these relations together with (3.6) give (3.1). Equality occurs in (3.1) when $p=H_{i \theta, \lambda}(z)$, for some $z \in E$. Conversely if equality occurs in (3.1) for some $z \in$ $E \backslash\{0\}$, then equality must hold in (3.3). Thus by Schwarz lemma there exists $\theta \in \mathbb{R}$ such that $w_{p}(z)=z \delta\left(e^{i \theta} z, \lambda\right)$ for all $z \in E$. This implies $P=H_{i \theta, \lambda}$.

Geometrically the above lemma means that the functional $p$ lies in the closed disk centred at $q(z, \lambda)$ with radius $r(z, \lambda)$.

For $\lambda=0$, we have the following result

Corollary 1. For $p \in P_{0}[A, B]$, we have

$$
\left|p(z)-\frac{1-A B|z|^{4}}{1-B^{2}|z|^{4}}\right| \leq \frac{(A-B)|z|^{2}}{1-B^{2}|z|^{4}}, z \in E \backslash\{0\} .
$$

Equality is attained if and only if $p=H_{i \theta, 0}$.

Corollary 2. Let $\gamma: z(t), 0 \leq t \leq 1$, be a $C^{1}$-curve in $E$ with $z(0)=0$ and $z(1)=z_{0}$. Then

$$
V_{\lambda}\left(z_{0}, A, B\right) \subset\{w \in \mathbb{C}:|w-Q(\lambda, \gamma)| \leq R(\lambda, \gamma)\},
$$

where

$$
Q(\lambda, \gamma)=\int_{0}^{1} q(z(t), \lambda) z^{\prime}(t) d t, \quad R(\lambda, \gamma)=\int_{0}^{1} r(z(t), \lambda) z^{\prime}(t) d t .
$$

Proof. Since $p$ is in $P_{\lambda}[A, B]$, therefore by using Proposition 2, we get

$$
\begin{aligned}
\left|\int_{0}^{1} p(z(t)) z^{\prime}(t) d t-Q(\lambda, \gamma)\right| & =\left|\int_{0}^{1} p(z(t)) z^{\prime}(t) d t-\int_{0}^{1} q(z(t), \lambda) z^{\prime}(t) d t\right| \\
& =\left|\int_{0}^{1}(p(z(t))-q(z(t), \lambda)) z^{\prime}(t) d t\right| \\
& \leq \int_{0}^{1} r(z(t), \lambda)\left|z^{\prime}(t)\right| d t=R(\lambda, \gamma) .
\end{aligned}
$$

This implies the required result.

For our next result we need the following lemma: 
Lemma 1. For $\theta \in \mathbb{R}$ and $|\lambda|<1$, the function

$$
G(z)=\int_{0}^{z} \frac{e^{i \theta} \zeta^{2}}{\left(1+\left(\bar{\lambda} e^{i \theta}+B \lambda\right) \zeta+B e^{i \theta} \zeta^{2}\right)^{2}} d \zeta, \quad z \in E,
$$

has zeros of order 3 at the origin and no zero elsewhere in E. Moreover, there exists a starlike normalized univalent function $s$ in $E$ such that $G(z)=3^{-1} e^{i \theta} s^{3}(z)$.

The above lemma is due to Ponnusamy et al. [4].

Proposition 3. Let $\theta \in(-\pi, \pi], z_{0} \in E \backslash\{0\}$. Then, $\int_{0}^{z_{0}} H_{e^{i \theta}, \lambda}(\rho) d \rho \in \partial V_{\lambda}\left(z_{0}, A, B\right)$. Moreover, $\int_{0}^{z_{0}} p(\rho) d \rho=\int_{0}^{z_{0}} H_{e^{i \theta}, \lambda}(\rho) d \rho$ implies $p=H_{e^{i \theta}, \lambda}$ for some $p \in P_{\lambda}[A, B]$ and $\theta \in(-\pi, \pi]$.

Proof. It follows from (2.1) that

$$
\begin{aligned}
H_{a, \lambda}(z) & =\frac{1+A z \delta(a z, \lambda)}{1+B z \delta(a z, \lambda)} \\
& =\frac{1+(\bar{\lambda} a+A \lambda) z+A a z^{2}}{1+(\bar{\lambda} a+B \lambda) z+B a z^{2}} .
\end{aligned}
$$

Thus from (3.5), it follows that

$$
\begin{aligned}
& H_{a, \lambda}(z)-b(z, \lambda)=\frac{(A-B)\left(1-|\lambda|^{2}\right) a z^{2}}{\left(1+(\bar{\lambda} a+B \lambda) z+B a z^{2}\right)(1+\lambda B z)}, \\
& H_{a, \lambda}(z)+c(z, \lambda)=\frac{(B-A)\left(1-|\lambda|^{2}\right) z}{\left(1+(\bar{\lambda} a+B \lambda) z+B a z^{2}\right)(B z+\bar{\lambda})} .
\end{aligned}
$$

Therefore

$$
\begin{aligned}
& H_{a, \lambda}(z)-q(z, \lambda) \\
& =H_{a, \lambda}(z)-\frac{b(z, \lambda)+|z|^{2}|\tau(z, \lambda)|^{2} c(z, \lambda)}{1-|z|^{2}|\tau(z, \lambda)|^{2}} \\
& =\frac{1}{1-|z|^{2}|\tau(z, \lambda)|^{2}}\left[H_{a, \lambda}(z)-b(z, \lambda)-|z|^{2}|\tau(z, \lambda)|^{2}\left(H_{a, \lambda}(z)+c(z, \lambda)\right)\right] \\
& =\frac{(A-B)\left(1-|\lambda|^{2}\right)\left[a z(1+B \overline{\lambda z})+|z|^{2}(B \bar{z}+\lambda)\right]}{\left(\begin{array}{c}
1-B^{2}|z|^{4}+2 B\left(1-|z|^{2}\right) \Re(\lambda z) \\
+|\lambda|^{2}|z|^{2}\left(B^{2}-1\right)
\end{array}\right)\left(1+(\bar{\lambda} a+B \lambda) z+B a z^{2}\right)} .
\end{aligned}
$$

Putting $a=e^{i \theta}$, we obtain

$$
H_{e^{i \theta, \lambda}}(z)-q(z, \lambda)
$$




$$
\begin{aligned}
& =\frac{r(z, \lambda) e^{i \theta} z^{2}}{|z|^{2}} \frac{\left(1+\left(\bar{\lambda} e^{i \theta}+B \lambda\right) z+B e^{i \theta} z^{2}\right) \overline{\left(1+\left(\bar{\lambda} e^{i \theta}+B \lambda\right) z+B e^{i \theta} z^{2}\right)}}{\left(1+\left(\bar{\lambda} e^{i \theta}+B \lambda\right) z+B e^{i \theta} z^{2}\right)^{2}} \\
& =\frac{r(z, \lambda) e^{i \theta} z^{2}}{|z|^{2}} \frac{\left|1+\left(\bar{\lambda} e^{i \theta}+B \lambda\right) z+B e^{i \theta} z^{2}\right|^{2}}{\left(1+\left(\bar{\lambda} e^{i \theta}+B \lambda\right) z+B e^{i \theta} z^{2}\right)^{2}} .
\end{aligned}
$$

Now using $G(z)$ defined in Lemma 1, it follows that

$$
H_{e^{i \theta}, \lambda}(z)-q(z, \lambda)=r(z, \lambda) \frac{G^{\prime}(z)}{\left|G^{\prime}(z)\right|} .
$$

Using the argument of Lemma 1 that $G=3^{-1} e^{i \theta} s^{3}$, where $s$ is starlike in $E$ with $s(0)=s^{\prime}(0)-1=0$, for any $z_{0} \in E \backslash\{0\}$ the linear segment joining 0 and $s\left(z_{0}\right)$ lies entirely in $s(E)$. Let $\gamma_{0}$ be the curve defined by

$$
\gamma_{0}: z(t)=s^{-1}\left(t s\left(z_{0}\right)\right), t \in[0,1] .
$$

Since $G(z(t))=2^{-1} e^{i \theta}(s(z(t)))^{2}=3^{-1} e^{i \theta}\left(t s\left(z_{0}\right)\right)^{3}=t^{3} G\left(z_{0}\right)$. Differentiation w.r.t $t$ gives us

$$
G^{\prime}(z(t)) z^{\prime}(t)=3 t^{2} G\left(z_{0}\right), \quad t \in[0,1] .
$$

This relation together with (3.7), leads to

$$
\begin{aligned}
\int_{0}^{z_{0}} H_{e^{i \theta, \lambda}}(\rho) d \rho-Q\left(\lambda, \gamma_{0}\right) & =\int_{0}^{1}\left(H_{e^{i \theta}, \lambda}(z(t))-q(z(t), \lambda)\right) z^{\prime}(t) d t \\
& =\int_{0}^{1} r(z(t), \lambda) \frac{G^{\prime}(z(t)) z^{\prime}(t)}{\left|G^{\prime}(z(t)) z^{\prime}(t)\right|}\left|z^{\prime}(t)\right| d t \\
& =\frac{G\left(z_{0}\right)}{\left|G\left(z_{0}\right)\right|} \int_{0}^{1} r(z(t), \lambda)\left|z^{\prime}(t)\right| d t \\
& =\frac{G\left(z_{0}\right)}{\left|G\left(z_{0}\right)\right|} R\left(\lambda, \gamma_{0}\right) .
\end{aligned}
$$

This implies that $\int_{0}^{z_{0}} H_{e^{i \theta}, \lambda}(\rho) d \rho \in \partial \bar{E}\left(Q\left(\lambda, \gamma_{0}\right), R\left(\lambda, \gamma_{0}\right)\right)$, where $Q\left(\lambda, \gamma_{0}\right)$ and $R\left(\lambda, \gamma_{0}\right)$ are defined as in Corollary 2. Hence from Corollary 2, we have $\int_{0}^{z_{0}} H_{e^{i \theta}, \lambda}(\rho) d \rho \in \partial V_{\lambda}\left(z_{0}, A, B\right)$. For uniqueness, we suppose that

$$
\int_{0}^{z_{0}} p(\rho) d \rho=\int_{0}^{z_{0}} H_{e^{i \theta}, \lambda}(\rho) d \rho
$$

for some $\theta \in(-\pi, \pi]$ and $p \in P_{\lambda}[A, B]$. Let

$$
h(t)=\frac{\overline{G\left(z_{0}\right)}}{\left|G\left(z_{0}\right)\right|}(p(z(t))-q(z(t), \lambda)) z^{\prime}(t),
$$


where $\gamma_{0}: z(t), 0 \leq t \leq 1$. Then the function $h$ is continuous and

$$
|h(t)|=\frac{\left|\overline{G\left(z_{0}\right)}\right|}{\left|G\left(z_{0}\right)\right|}|(p(z(t))-q(z(t), \lambda))|\left|z^{\prime}(t)\right| .
$$

Now using Proposition 2, we get $|h(t)| \leq r(z(t), \lambda)\left|z^{\prime}(t)\right|$. Further from (3.9), we have

$$
\begin{aligned}
\int_{0}^{1} \Re(h(t)) d t & \left.=\int_{0}^{1} \Re\left(\frac{\overline{G\left(z_{0}\right)}}{\left|G\left(z_{0}\right)\right|}(p(z(t))-q(z(t), \lambda)) z^{\prime}(t)\right]\right) d t \\
& =\Re\left[\frac{\overline{G\left(z_{0}\right)}}{\left|G\left(z_{0}\right)\right|} \int_{0}^{z_{0}}\left\{H_{e^{i \theta}, \lambda}(\rho) d \rho-Q(z(t), \lambda)\right\}\right] \\
& =\int_{0}^{1} \Re(r((z(t), \lambda)))\left|z^{\prime}(t)\right| d t .
\end{aligned}
$$

Thus $h(t)=r(z(t), \lambda)\left|z^{\prime}(t)\right|$, for all $t \in[0,1]$. From (3.7) and (3.8) we have $\int_{0}^{z_{0}} p(\rho) d \rho=\int_{0}^{z_{0}} H_{e^{i \theta}, \lambda}(\rho) d \rho$ on $\gamma_{0}$. The identity theorem for analytic functions yields us $p=H_{e^{i \theta}, \lambda}, z \in E$.

\section{Main Theorem}

Theorem 1. Let $\lambda \in E$ and $z_{0} \in E \backslash\{0\}$. Then boundary $\partial V_{\lambda}\left(z_{0}, A, B\right)$ is the Jordan curve given by

$$
(-\pi, \pi] \ni \theta \mapsto \int_{0}^{z_{0}} H_{e^{i \theta}, \lambda}(\rho) d \rho=\int_{0}^{z_{0}} \frac{1+A \rho \delta\left(e^{i \theta} \rho, \lambda\right)}{1+B \rho \delta\left(e^{i \theta} \rho, \lambda\right)} d \rho
$$

If $\int_{0}^{z_{0}} p(\rho) d \rho=\int_{0}^{z_{0}} H_{e^{i \theta}, \lambda}(\rho) d \rho$ for some $p \in P_{\lambda}[A, B]$ and $\theta \in(-\pi, \pi]$, then $p(z)=H_{e^{i \theta}, \lambda}(z)$.

Proof. First we have to show that the curve

$$
(-\pi, \pi] \ni \theta \mapsto \int_{0}^{z_{0}} H_{e^{i \theta}, \lambda}(\rho) d \rho
$$

is simple. Let us assume that

$$
\int_{0}^{z_{0}} H_{e^{i \theta_{1}, \lambda}}(\rho) d \rho=\int_{0}^{z_{0}} H_{e^{i \theta_{2}, \lambda}}(\rho) d \rho
$$

for some $\theta_{1}, \theta_{2} \in(-\pi, \pi]$ with $\theta_{1} \neq \theta_{2}$. Then the use of Proposition 3 yields us that $H_{e^{i \theta_{1}, \lambda}}\left(z_{0}\right)=H_{e^{i \theta_{2}, \lambda}}\left(z_{0}\right)$, which further gives the following relation

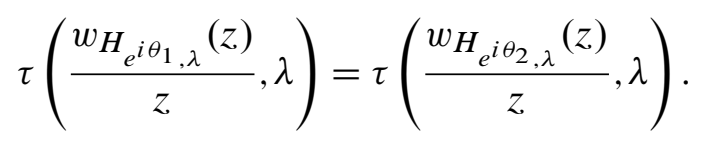


This implies that

$$
\frac{B\left(z e^{i \theta_{1}}+\lambda\right)+\bar{\lambda}\left(1+\bar{\lambda} e^{i \theta_{1}} z\right)}{1+\bar{\lambda} e^{i \theta_{1}} z+\lambda B\left(z e^{i \theta_{1}}+\lambda\right)}=\frac{B\left(z e^{i \theta_{2}}+\lambda\right)+\bar{\lambda}\left(1+\bar{\lambda} e^{i \theta_{2}} z\right)}{1+\bar{\lambda} e^{i \theta_{2}} z+\lambda B\left(z e^{i \theta_{2}}+\lambda\right)} .
$$

After some simplification, we obtain $z e^{i \theta_{1}}=z e^{i \theta_{2}}$, which leads us to a contradiction. Hence the curve is simple. Since $V_{\lambda}\left(z_{0}, A, B\right)$ is compact convex subset of $\mathbb{C}$ and has non-empty interior, therefore the boundary $\partial V_{\lambda}\left(z_{0}, A, B\right)$ is a simple closed curve. From Proposition 3 the curve $\partial V_{\lambda}\left(z_{0}, A, B\right)$ contains the curve $(-\pi, \pi] \ni$ $\theta \mapsto \int_{0}^{z_{0}} H_{e^{i \theta}, \lambda}(\rho) d \rho$. Since a simple closed curve cannot contain any simple closed curve other than itself. Thus $\partial V_{\lambda}\left(z_{0}, A, B\right)$ is given by $(-\pi, \pi] \ni \theta \mapsto$ $\int_{0}^{z_{0}} H_{e^{i \theta}, \lambda}(\rho) d \rho$.

\section{Geometric View of Theorem 1}

By using Mathematica, we have the following views for different parameters. 


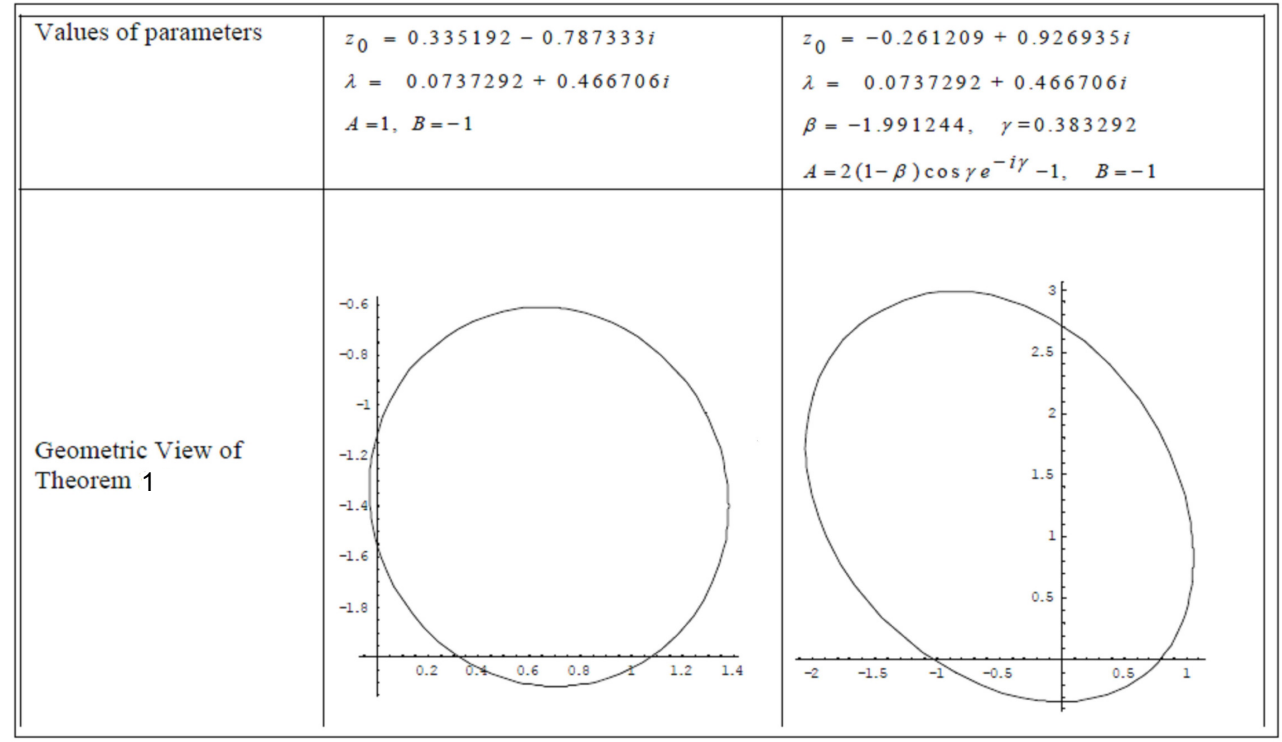

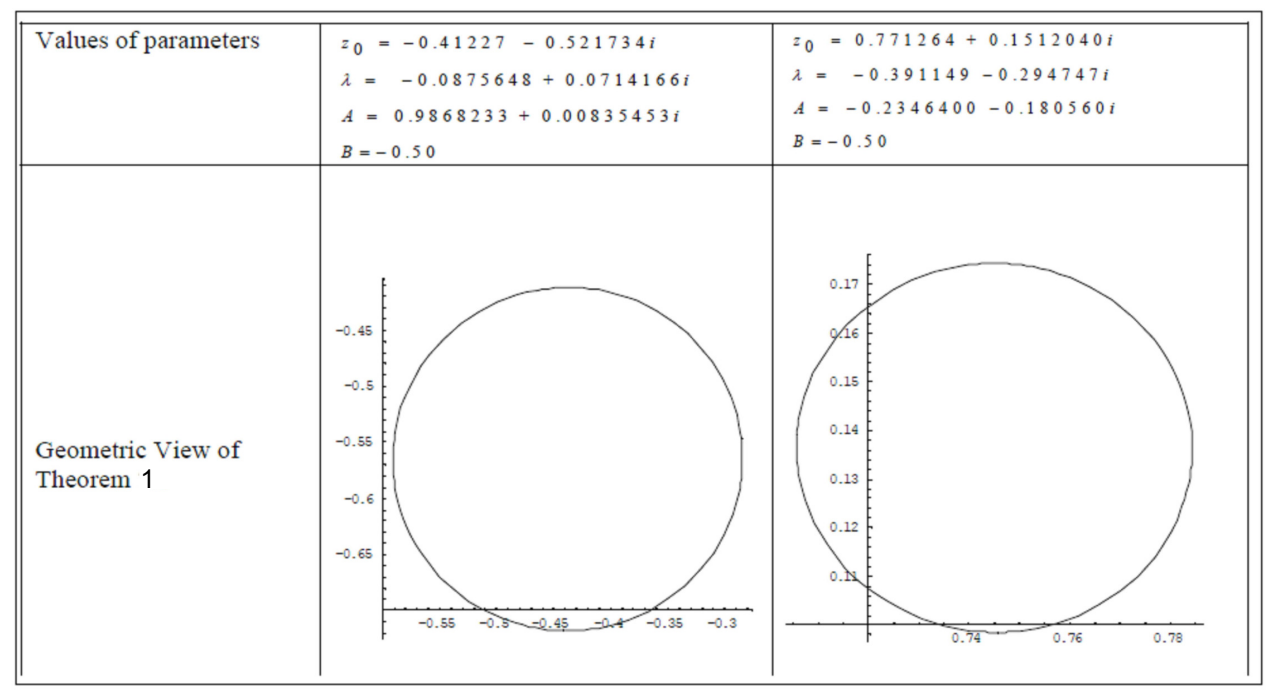

FIGURE 1. For $A=2(1-\beta)(\cos \gamma) e^{-i \gamma}-1, B=-1$, where $\beta<$ $1,|\gamma|<\pi / 2$, we have the known result proved by Ponnusamy and Vasudevarao [3] as special cases of our results. 


\section{REFERENCES}

[1] S. Dineen, The Schwarz lemma, ser. Oxford Mathematical Monographs. Oxford: Clarendon Press, 1989.

[2] S. Ponnusamy and A. Vasudevarao, "Region of variability of two subclasses of univalent functions," J. Math. Anal. Appl., vol. 332, no. 2, pp. 1323-1334, 2007, doi: 10.1016/j.jmaa.2006.11.019.

[3] S. Ponnusamy and A. Vasudevarao, "Region of variability for functions with positive real part," Ann. Polon. Math., vol. 99, no. 3, pp. 225-245, 2010, doi: 10.4064/ap99-3-2.

[4] S. Ponnusamy, A. Vasudevarao, and M. Vuorinen, "Region of variability for certain classes of univalent functions satisfying differential inequalities," Complex var. Elliptic Equ., vol. 54, no. 10, pp. 899-922, 2009, doi: 10.1080/17476930802657616.

[5] S. Ponnusamy, A. Vasudevarao, and H. Yanagihara, "Region of variability for close-toconvex functions," Complex Var. Elliptic Equ., vol. 53, no. 8, pp. 709-716, 2008, doi: 10.1080/17476930801996346.

[6] S. Ponnusamy, A. Vasudevarao, and H. Yanagihara, "Region of variability of univalent functions $\mathrm{f}(\mathrm{z})$ for which zf'(z) is spirallike," Houston J. Math., vol. 34, no. 4, pp. 1037-1048, 2008.

[7] W. Ul-Haq, "Variability regions for janowski convex functions," Complex Var. Elliptic Equ., vol. 59, no. 3, pp. 355-361, 2014, doi: 10.1080/17476933.2012.725164.

[8] H. Yanagihara, "Regions of variability for functions of bounded derivatives," Kodai Math. J., vol. 28, no. 2, pp. 452-462, 2005, doi: 10.2996/kmj/1123767023.

[9] H. Yanagihara, "Regions of variability for convex functions," Math. Nachr., vol. 279, no. 15, pp. 1723-1730, 2006, doi: 10.1002/mana.200310449.

Authors' addresses

M. Raza

Department of Mathematics, Government College University Faisalabad, Pakistan

E-mail address: mohsan976@yahoo.com

\section{W. Ul-Haq}

Department of Mathematics, College of Science in Al-Zulfi, Majmaah University, Al-Zulfi, Saudi Arabia

E-mail address: wasim474@hotmail.com

\section{S. Noreen}

Department of Mathematics, Government College University Faisalabad, Pakistan

E-mail address: sadda fnoreeneyahoo.com 